\title{
Simulation of the Linear Boltzmann Transport Equation in Modelling Of Photon Beam Data
}

\author{
Akpochafor M. O., Aweda M. A., Durosinmi-Etti F.A., Adeneye S.O., \\ Omojola AD. \\ Department of Radiation Biology, Radiotherapy, Radiodiagnosis and Radiography, \\ College of Medicine/Lagos University Teaching Hospital, PMB 12003, Lagos, Nigeria.
}

\begin{abstract}
A beam data modelling algorithm was developed by solving the linear Boltzmann Transport Equation (BTE). The Linear Boltzmann Transport Equation (LBTE) is a form of the Boltzmann transport equation that assumes that radiation particles only interact with the matter as they are passing through matter and not with each other. This condition is only valid when there is no external magnetic field. The numerical method proposed by Lewis et al., [9] was used to solve the LBTE. A programming code was computed for the LBTE and run on CMS XiO treatment planning system to generate beam data, the generated beam data were compared to experimentally determined data. The calculated percentage depth dose (PDD) completely overlap the measured PDDs for the small field sizes while there is a shift in the PDD tail for large field size. However the shift is negligible. For the wedge PDDs, the shift between the measured PDDs and the calculated occurs at the $D_{\max }$ region and it increases with increase in field size. The calculated wedge profiles have a slight shift at the shoulder compared to the measured ones and this decreases with increase in field size, unlike the PDDs. There is also a slight shift between calculated in-plane profiles and measured ones. There is a good agreement between the measured beam data and the calculated ones using the algorithm. This algorithm can be implemented as an in-house algorithm for beam data modelling and also as an independent quality assurance tool for checking the accuracy of clinical TPS algorithms with regards to beam data modelling during quality assurance and TPS commissioning tests.
\end{abstract}

Keywords: linear Boltzmann Transport Equation (BTE), treatment planning system, algorithm, beam profile, percentage depth dose.

\section{Introduction}

Computerized Treatment Planning Systems (TPS) are used in external beam radiotherapy to simulate beam shapes and dose distribution with the intent to optimize tumour control and minimize normal complications [7]. Treatment simulations involve the geometric and radiological aspects of the treatment and it is based on radiation transport and optimization principles. TPS facilitate prescribed dose delivery in which a number of the patient data and of the tumour parameters have to be taken into consideration such as the shape, size, depth etc. Following acquisition of a new TPS, it is necessary to perform the commissioning tests, a process which involves the entry of beam data measured at the linear accelerator into the TPS for precise modelling of the dose distribution. Beam profiles and Percentage depth doses (PDD) are some of the most important beam characteristics required for the commissioning of the TPS. The profiles and the PDD combine to form the isodose curves which determine the dose distribution in the treatment plan of a radiotherapy patient. The profile tails also determine the penumbra size of the dose distribution which plays a significant role in the total dose distribution. An improvement in the penumbra size of the profile will lead to a better dose distribution. The modelling of the beam data is done using the TPS software. The accuracy of the model depends on the software parameters [3]. There are several algorithms contained in the TPS software that play different roles, the dose calculation algorithms among these play the central role of calculating dose distribution within the target volume [1]. Algorithms are a sequence of instructions that use a set of input patient and dosimetric data to transforming the information into a set of desired output results [8]. For every algorithm, the precision of the dose calculation depends on the input parameters. Different types of dose calculation algorithms are used in modern TPS. The early TPS calculation methods were based on tabular representation of the dose distribution obtained directly from beam measurements. As time went by, calculation models become more sophisticated as computation power grew. TPS calculation algorithms progressively matured towards more physically based models. The most advanced current algorithms are based on the Monte Carlo approach where the histories of many millions of photons are traced as they interact with matter using basic physics interactions. There is a full range of possibilities between table-based models and Monte Carlo models. For every algorithm, the quality of the dose calculation is strongly dependent on the data or parameters used by the algorithm and its accuracy to predict dose rely on the assumptions and approximations that the algorithm makes. The type and 
quantity of the data needed varies according to the model. Usually, for measurement based models a lot of tables are required, whereas for physical based models only some parameters are necessary. Good understanding of the algorithms used within the TPS can help the user understand the strength and limitations of the particular algorithm. This can also help the user diagnose TPS problems and develop a quality assurance (QA) protocol. It is important to understand the general principles of the model and its implementation details. The model parameters and input data have a significant impact on the accuracy of the calculated results. Even if the model is able to account for a given physical effect, the actual implementation in the treatment planning software is often simplified, leading to inaccurate or unexpected results in certain situations. Because of these situations an independent way of checking the algorithms accuracy in beam modelling is vital to achieve a proper QA exercise. Following the acceptance and commissioning tests of a computerized TPS, a quality assurance program should be established to verify the performance of the system. Several ways of carrying out the quality assurance has been proposed in the literature [1-6]. It is necessary that each Department develop its own protocol based on the availability of relevant equipment and according to local requirements, using standard methods as guideline.

In this study, the linear Boltzmann transport equation (LBTE) was solved following the numerical methods described by Lewis et al. [9], a programming code was developed for the LBTE and run on a CMS $\mathrm{XiO}$ treatment planning system to generate beam profiles and PDD. The generated beam data were compared with experimentally measured and analyzed data.

\section{Methods And Material}

The Boltzmann transport equation (BTE) is the governing equation which describes the macroscopic behaviour of radiation particles like photons, electrons, neutrons, protons, etc. as they travel through and interact with matter. The Linear Boltzmann transport equation (LBTE) is a form of the BTE which assumes that radiation particles only interact with the matter they are passing through and not with each other. This is valid for conditions in the absence of external magnetic fields. There are different ways of solving the LBTE, however, the numerical method proposed by Lewis et al., [9] is the method of choice for solving the equation explicitly. The LTBE was solved using a similar method by Vassiliev et al. [15]:

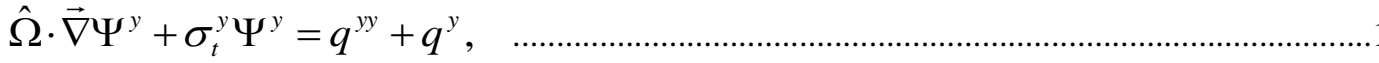

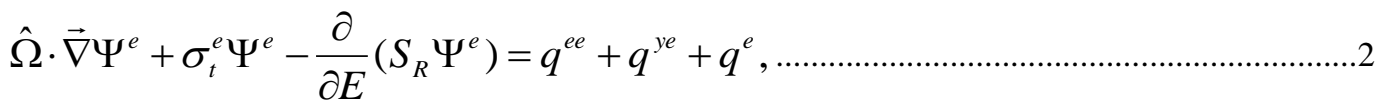

where $\quad \Psi^{y}=$ Angular photon fluence (or fluence if not time integrated),

$\Psi^{y}(\overrightarrow{\mathrm{r}}, E, \hat{\Omega})$, is a function of position $\vec{r}=(\mathrm{x}, \mathrm{y}, \mathrm{z})$, energy $\mathrm{E}$, and direction $\hat{\Omega}=(\mu, \eta, \zeta)$

$\Psi^{e}=$ Angular electron fluence $\Psi^{\gamma}(\overrightarrow{\mathrm{r}}, E, \hat{\Omega})$

$q^{y y}=$ Photon - photon scattering source, $q^{y y}(\overrightarrow{\mathrm{r}}, E, \hat{\Omega})$, resulting from photon interactions.

$q^{e e}=$ Election - electron scattering source, $q^{e e}(\overrightarrow{\mathrm{r}}, E, \Omega)$, resulting from electron interactions.

$q^{y e}=$ Photon- electron scattering source, $q^{y e}(\overrightarrow{\mathrm{r}}, E, \hat{\Omega})$, resulting from electron interactions.

$q^{y}=$ Extraneous photon source, $q^{y}(E, \hat{\boldsymbol{\Omega}})$, for point source $\boldsymbol{P}$, at position $\overrightarrow{\mathrm{r}}_{\mathrm{p}}$

This source represents all photons coming from the machine source model (Wareing et al., 2000).

$q^{e}=$ Extraneous electron source, $q^{y}(E, \hat{\Omega})$, for point source $\boldsymbol{P}$, as position $\overrightarrow{\mathrm{r}}_{\mathrm{p}}$

This source represents all electrons coming from the machine source model.

$\sigma_{t}^{y}=\sigma_{t}^{y}(\overrightarrow{\mathrm{r}}, E)$ is the macroscopic photon total cross section in $\mathrm{cm}^{-1}$

$\sigma_{t}^{e}=\sigma_{t}^{e}(\overrightarrow{\mathrm{r}}, E)$ is the macroscopic electron total cross section in $\mathrm{cm}^{-1}$

$\sigma_{t}=\sigma_{t} \quad(\overrightarrow{\mathrm{r}}, E)$, is the macroscopic total cross section in $\mathrm{cm}^{-1}$

$\mathrm{S}_{\mathrm{R}}=\mathrm{S}_{\mathrm{R}}(\overrightarrow{\mathrm{r}}, E)$ is the restricted collisional plus radiative stopping power,

The first term on the left hand side of equations 1 and 2 is the streaming operator. The second term on the left hand side of equations 1 and 2 is the collision or removal operator. Equation 2 is the Boltzmann Fokker-Planck transport equation [11-12], which is solved for the electron transport. In Equation 2, the third term on the left 
represents the continuous slowing down (CSD) operator, which accounts for Coulomb 'soft' electron collisions. The right hand side of Equations 1 and 2 include the scattering, production, and the external source terms $\left(q^{y}\right.$ and $\left.q^{e}\right)$. The scattering and production sources are defined by:

$$
\begin{aligned}
& q^{y y}(\overrightarrow{\mathrm{r}}, E, \hat{\Omega}),=\int_{0}^{\infty} d E^{\prime} \int_{4 \pi} d \Omega^{\prime} \sigma_{s}^{y y}\left(\vec{r}, E^{\prime} \rightarrow E, \hat{\Omega} \cdot \hat{\Omega}^{\prime}\right) \Psi^{y}\left(\vec{r}, E^{\prime}, \hat{\Omega}^{\prime}\right), \\
& q^{y e}(\overrightarrow{\mathrm{r}}, E, \hat{\Omega}),=\int_{0}^{\infty} d E^{\prime} \int_{4 \pi} d \Omega^{\prime} \sigma_{s}^{y e}\left(\vec{r}, E^{\prime} \rightarrow E, \hat{\Omega} \cdot \hat{\Omega}^{\prime}\right) \Psi^{y}\left(\vec{r}, E^{\prime}, \hat{\Omega}^{\prime}\right), \\
& q^{e e}(\overrightarrow{\mathrm{r}}, E, \hat{\Omega}),=\int_{0}^{\infty} d E^{\prime} \int_{4 \pi} d \Omega^{\prime} \sigma_{s}^{e e}\left(\vec{r}, E^{\prime} \rightarrow E, \hat{\Omega} \cdot \hat{\Omega}^{\prime}\right) \Psi^{e}\left(\vec{r}, E^{\prime}, \hat{\Omega}^{\prime}\right),
\end{aligned}
$$

where $\sigma_{s}^{y y}=$ Macroscopic photon-to-photon differential scattering cross section

$\sigma_{s}^{y e}=$ Macroscopic photon-to-electron differential production cross section

$\sigma_{s}^{e e}=$ Macroscopic electron-to-electron differential scattering cross section.

The following equation 6 represents the un-collided photon fluence:

$\hat{\Omega} \cdot \vec{\nabla} \Psi_{u n c}^{y}+\sigma_{t}^{y} \Psi_{u n c}^{y}=q^{y}(E, \hat{\Omega}) \delta\left(\vec{r}-\vec{r}_{p}\right)$,

A property of Equation 6 was that $\vec{\nabla} \Psi_{u n c}^{y}$ can be solved for analytically. Doing so provides the following expression for the un-collided photon angular fluence from a point source:

$$
\Psi_{u n c}^{y}(\vec{r}, E, \hat{\Omega})=\delta\left(\hat{\Omega}-\hat{\Omega}_{\vec{r}, \vec{r}_{p}}\right) \frac{q^{y}(E, \hat{\Omega})}{4 \pi} \frac{e^{-\tau\left(\vec{r}, \vec{r}_{p}\right)}}{\left|\vec{r}-\vec{r}_{p}\right|^{2}},
$$

where, $\hat{\Omega}_{\vec{r}, \vec{r}_{p}}=\frac{\left|\vec{r}_{p}-\vec{r}\right|}{\left|\vec{r}-\vec{r}_{p}\right|}$, and $\vec{r}_{p}$ and $\vec{r}$ are the source and destination points of the ray trace, respectively. $\tau\left(\vec{r}-\vec{r}_{p}\right)$ is the optical distance (measured in mean-free-paths) between $\vec{r}$ and $\vec{r}_{p}$.

Once the electron angular fluence was solved for all energy groups, the dose in any output grid voxel was obtained through the following equation proposed by Siebers et al. [13]:

$D_{i}=\int_{0}^{\infty} d E \int_{4 \pi} d \hat{\Omega} \frac{\sigma_{E D}^{e}(\vec{r}, E)}{\rho(\vec{r})} \Psi^{e}(\vec{r}, E, \vec{\Omega})$

where $\sigma_{E D}^{e}=$ macroscopic electron energy deposition cross sections (in $\mathrm{MeV} / \mathrm{cm}$ )

$\rho=$ Material density $\left(\right.$ in $\left.\mathrm{g} / \mathrm{cm}^{3}\right)$.

The iteration scheme used in solving the equation is shown in figure 1 below.

\section{Experimental determination of radiation beam profiles and PDDs}

A pre-calibrated Eleckta precise clinical linear accelerator was used to collect the beam data (profile and PDD). The profile and the PDD data were collected by following the guideline recommended by the CMS $\mathrm{XiO}$ beam modelling guide [14]. The diagonal profile scans were collected at an SSD of value of $100 \mathrm{~cm}$ with the largest open field size $40 \times 40 \mathrm{~cm}^{2}$ and at various depths of $0.5,1.0,2.0,3.0,5.0,10.0,20.0,30.0$, up to the deepest obtainable depth in $\mathrm{cm}$. Scans were generated at an increment depth of $3 \mathrm{~mm}$. The open field profiles were collected for the square field sizes of $5 \times 5$ and $30 \times 30 \mathrm{~cm}^{2}$ at depths of $\mathrm{d}_{\max }, 5.0,10.0,20.0$, and $30.0 \mathrm{~cm}$. Scans were made in the in-plane direction for fixed collimator. Scans were made at an increment depth of $2 \mathrm{~mm}$. Wedge aligned profile scans were collected in the wedge direction for the square field sizes of $10 \mathrm{x} 10$ and $20 \mathrm{x}$ $20 \mathrm{~cm}^{2}$ at depths $\mathrm{d}_{\max }, 5.0,10.0$, and $20.0 \mathrm{~cm}$. The open field PDDs were measured at the field sizes of $3 \times 3$ and $30 \times 30 \mathrm{~cm}^{2}$. The scans were made at an increment depth of $1 \mathrm{~mm}$ up to the deepest obtainable depth of $35 \mathrm{~cm}$. The wedge field PDDs were measured at the field sizes of $5 \times 5,10 \times 10$ and $20 \times 20 \mathrm{~cm}^{2}$. Scans were also acquired at an increment depth of $1 \mathrm{~mm}$ up to the deepest obtainable depth. Once all scans were acquired for both 6 and $18 \mathrm{MeV}$ photon beams, they were compared with the computed ones using the algorithm above. The experimental set up for the measurement is shown in figure 2 below. 
Fig. 1: The iteration scheme used to solve the equations is shown in the algorithm below

\% File: Linearized Boltzmann Equations

$\%$ Date: 12th of March 2012

$\%$ Author: Michael Akpochafor

$\%$ The equation here perform time independent single calculation at high resolutions

$\% \mathrm{D}(\operatorname{vector}(\mathrm{r}))=\operatorname{lint}(\mathrm{mu} / \mathrm{P}) * \backslash(\mathrm{psi}) \_\mathrm{p}\left\{\operatorname{vector} \quad(\mathrm{r})^{\prime *} \mathrm{~A}^{*}\left[\operatorname{vector}(\mathrm{r})-\operatorname{vector}(\mathrm{r})^{\prime}\right] \mathrm{d}^{\wedge} 3\right.$ $\left.\left.*(\operatorname{vector}(\mathrm{r}))^{\prime}\right)\right\}$

$\% \mathrm{D}($ vector $(\mathrm{r}))=$ dose at a point

$\%(\mathrm{mu} / \mathrm{P})=$ mass attenuation coefficient

$\% \backslash(p s i) \_p\left\{\right.$ vector $(\mathrm{r})^{\prime}=$ primary photon energy fluence

$\% \mathrm{~A}^{*}\left[\operatorname{vector}(\mathrm{r})\right.$-vector$\left.(\mathrm{r}){ }^{\prime}\right]=$ convolution kernel, the distribution of fraction energy

Imparted per unit volume.

$\%$ (vector $\left.(\mathrm{r})^{\prime}\right)=$ TERMA at depth includes the energy retained by the photon.

$\%$ Plots a Linearized Boltzmann distribution Equations

$\%$ for dose calculation.

$\%$ THIS PROGRAMME SOLVE THE EQUATION (8)

$\% \mathrm{D}(\mathrm{i})=$ int_0 $\wedge^{\wedge}$ inffy $* \mathrm{dE} *$ int_4*pi ${ }^{\wedge}$ inffy $* \mathrm{~d}($ lomega) vector * (frac $\backslash$ sigma_ED

$\% \wedge$ e (r(vector), E)/rho(vector)*r(vector)* $\mid$ psi^e (r,E, lomega(all vector))

max=input('maximum time');

l=input('length');

stepx $=$ input $(' \mathrm{dx}=1$ ')

stept=input('dt=0.1')

$\operatorname{maxt}=10$;

$\mathrm{x}=0$ :stepx: 1 ;

$\mathrm{t}=0$ :stept:maxt;

$\mathrm{u}=\mathrm{zeros}($ length$(\mathrm{x})$,length $(\mathrm{t}))$

$\mathrm{r}=($ step$) /($ stepx $*$ stepx $)$;

$\mathrm{u}(1,:)=$ input('boundary temperature')*ones $(\operatorname{size}(\mathrm{t}))$;

for $\mathrm{j}=1$ :length(t)-1;

for $\mathrm{i}=2$ :length $(\mathrm{x})-1$;

$u(i, j+1)=r * u(i-1, j)+(1-2 * r)^{*} u(i, j)+r * u(i+1, j)$

end

end 


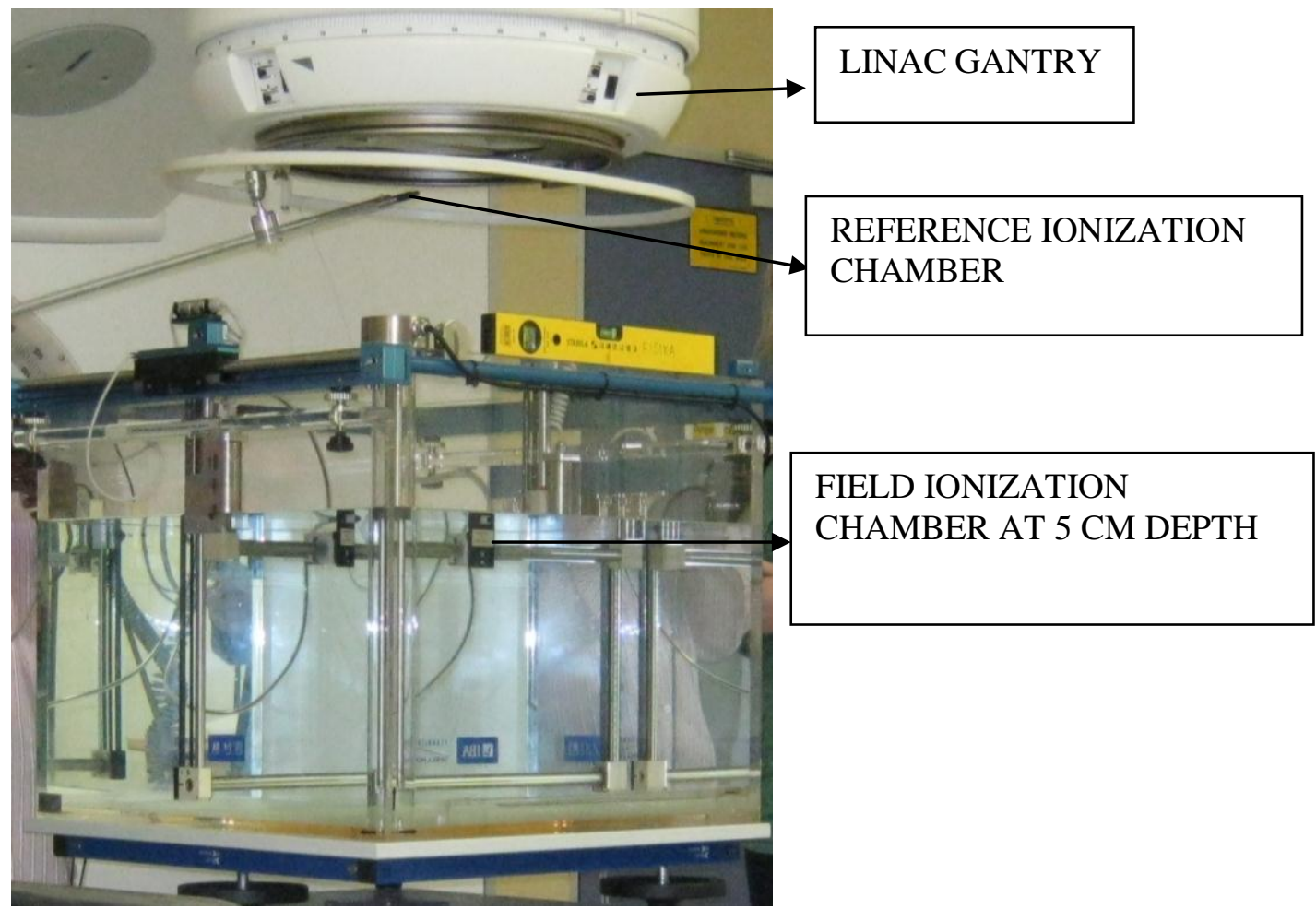

Fig. 2: Experimental set up for acquisition of beam profile and PDD scans.

Scanned data for $6 \mathrm{MeV}$ photon beam

\section{Results}

Below are the results of the measured vs calculated PDDs and profiles. The coloured lines

(_Lrepresents the calculated PDDs and profiles while the black line ( ) represents thamaasured ones.

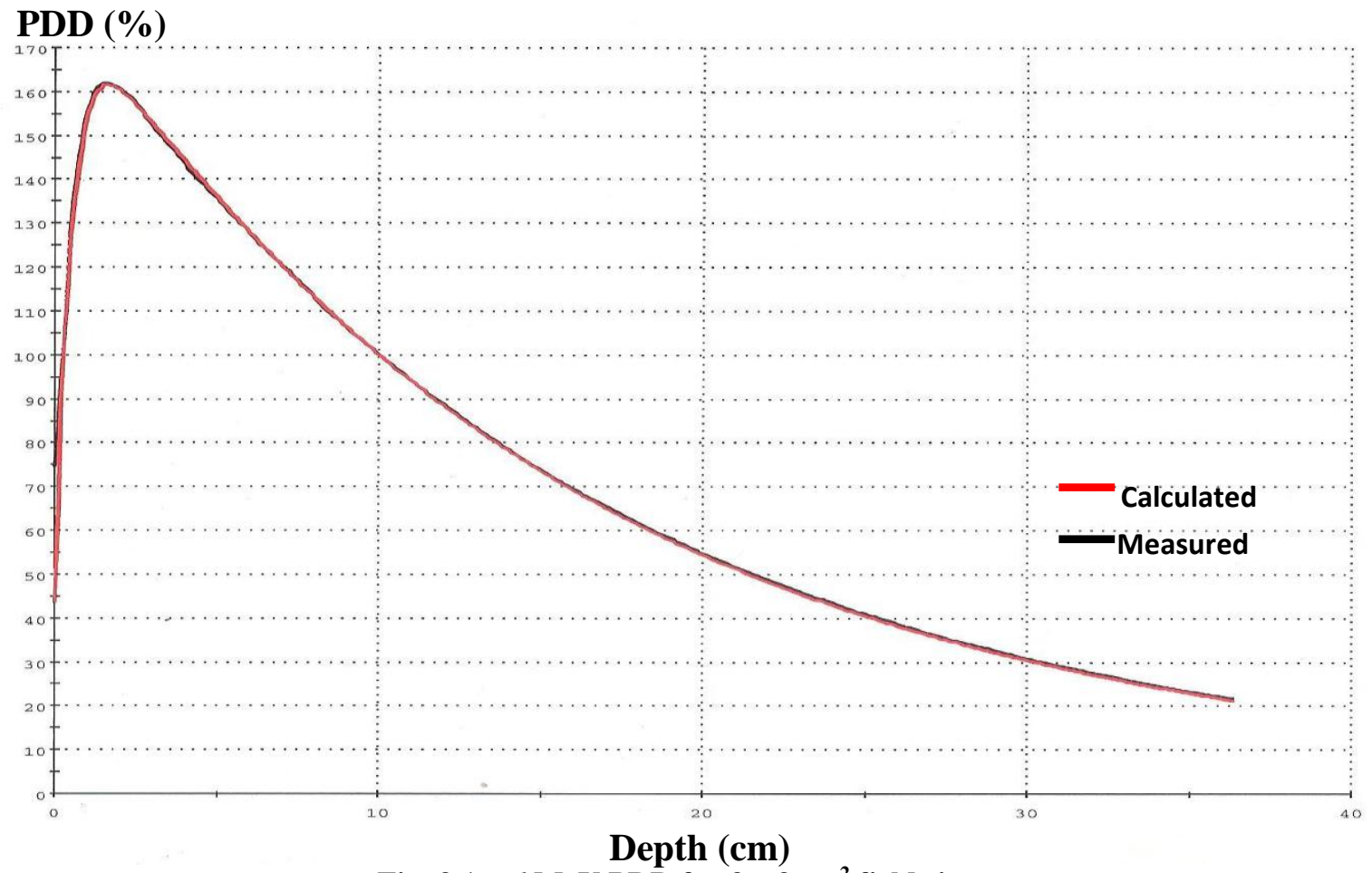

Fig. 3.1a: $6 \mathrm{MeV}$ PDD for $3 \times 3 \mathrm{~cm}^{2}$ field size 


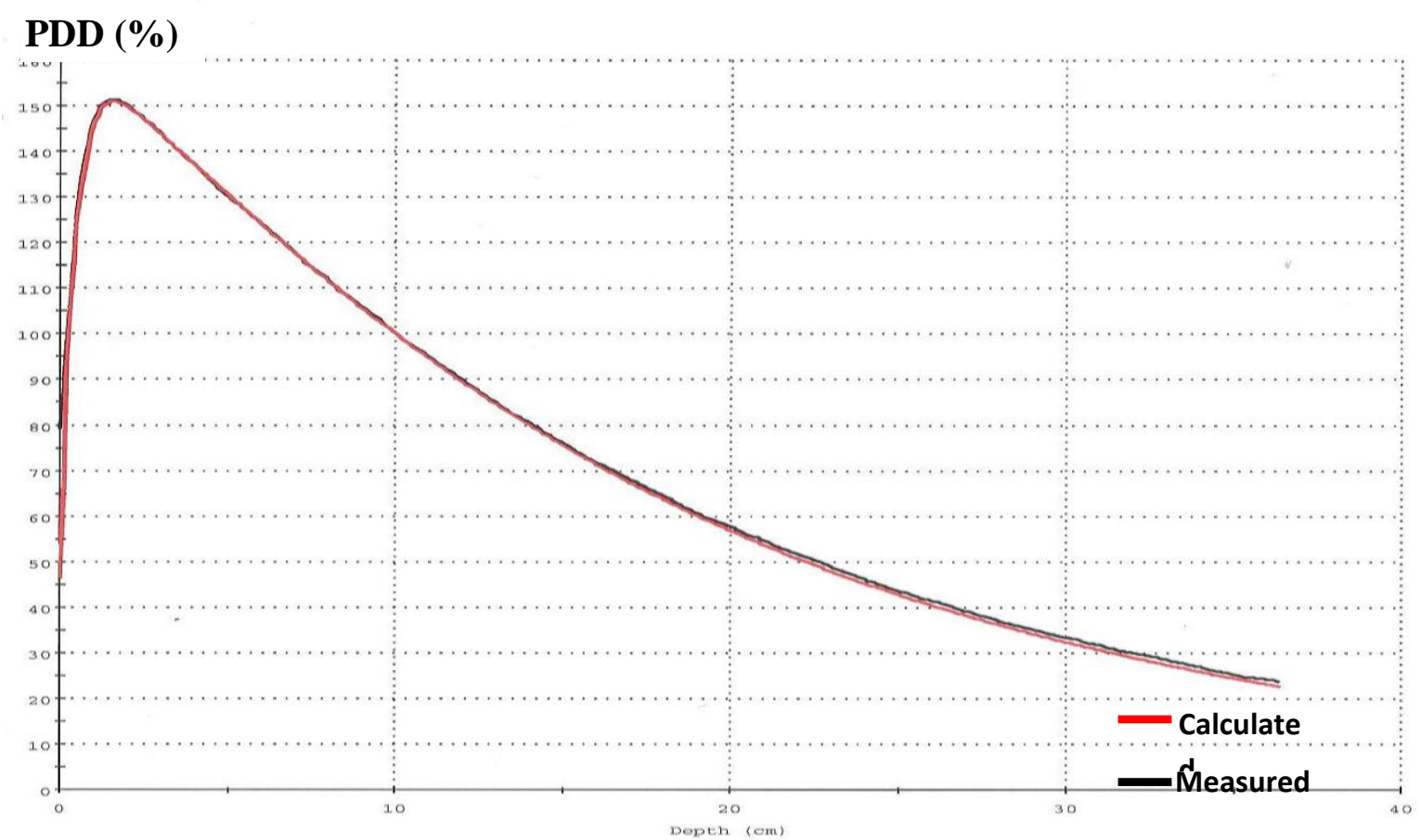

PDD (\%) Depth (cm) Fig. 3.1b: $6 \mathrm{MeV}$ PDD for $8 \times 8 \mathrm{~cm}^{2}$ field size

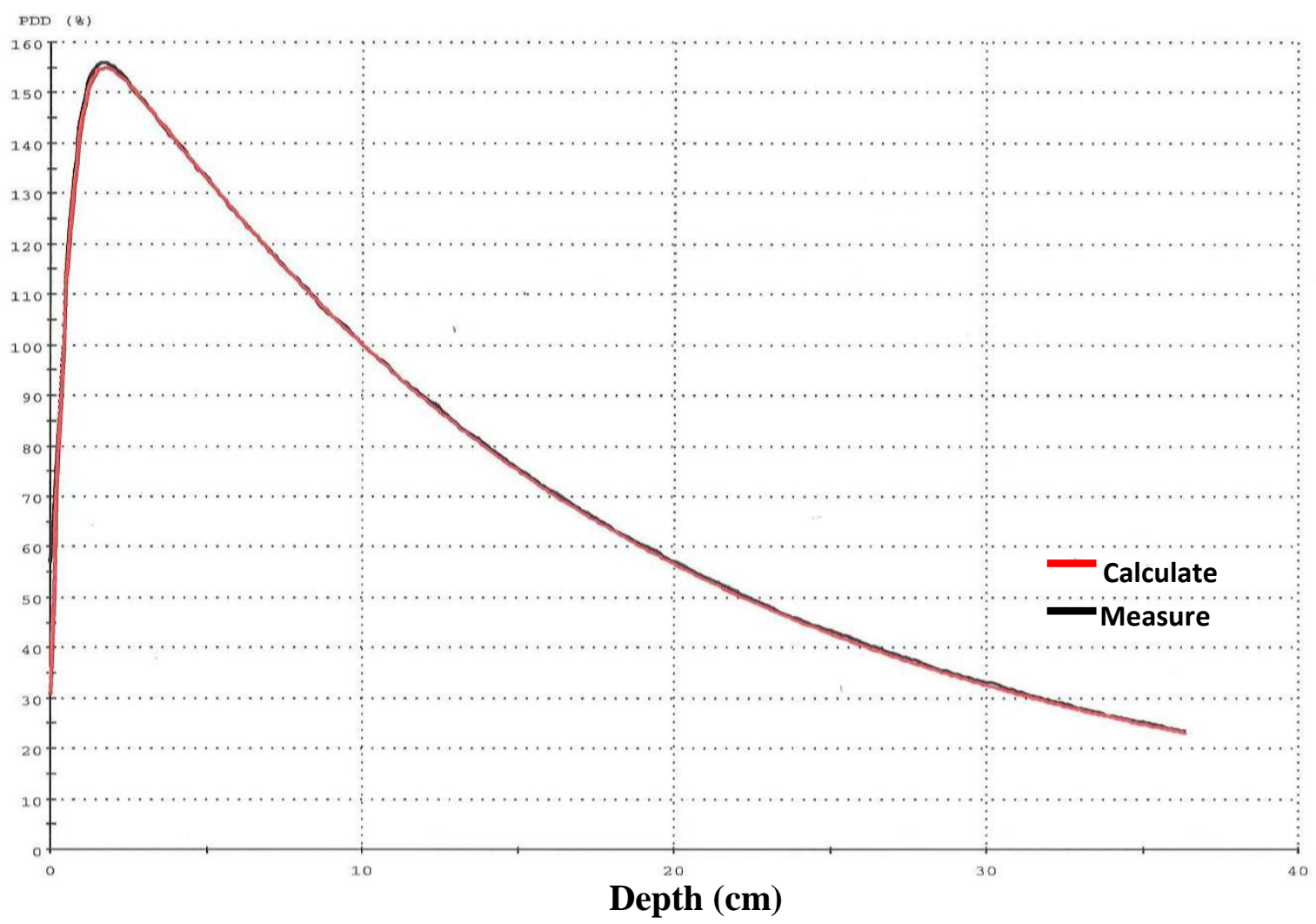

Fig. 3.2a: $6 \mathrm{MeV}$ wedge PDD for $3 \times 3 \mathrm{~cm}^{2}$ field size

PDD (\%)

Depth $(\mathbf{c m})$ 


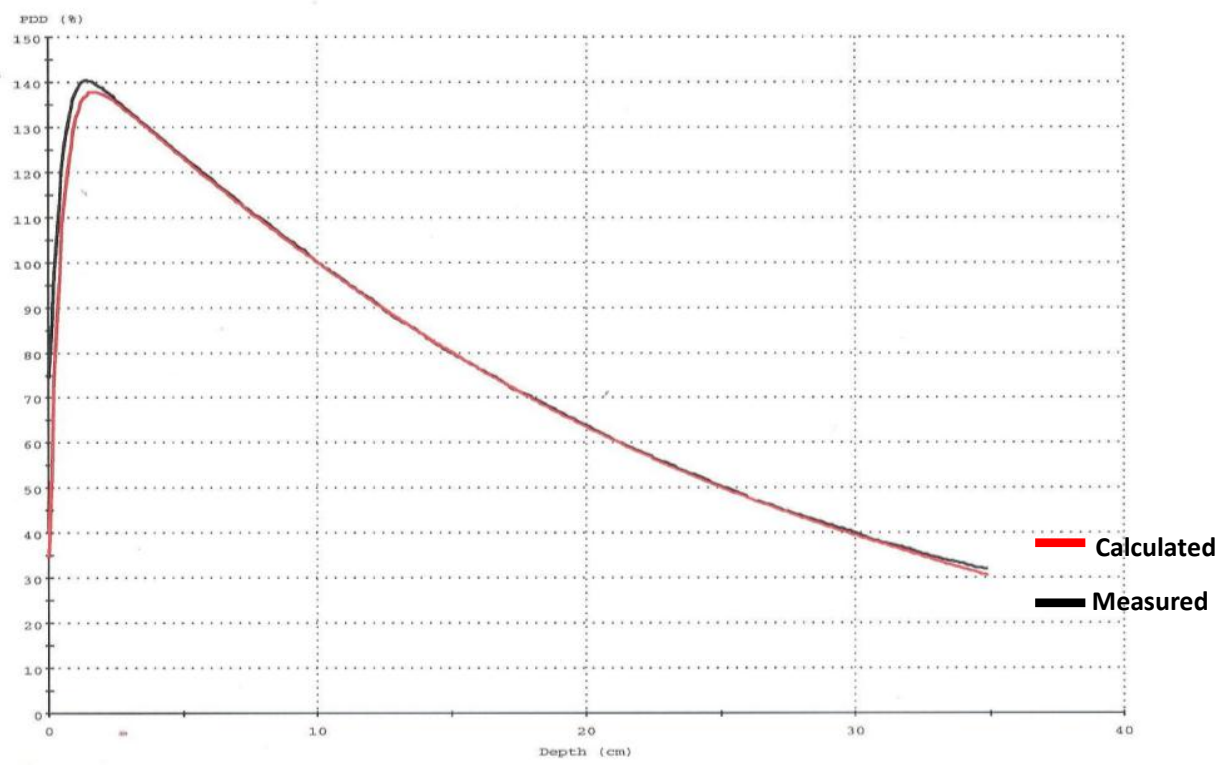

Fig. 3.2b: $6 \mathrm{MeV}$ wedge PDD for $3 \times 3 \mathrm{~cm}^{2}$ field size

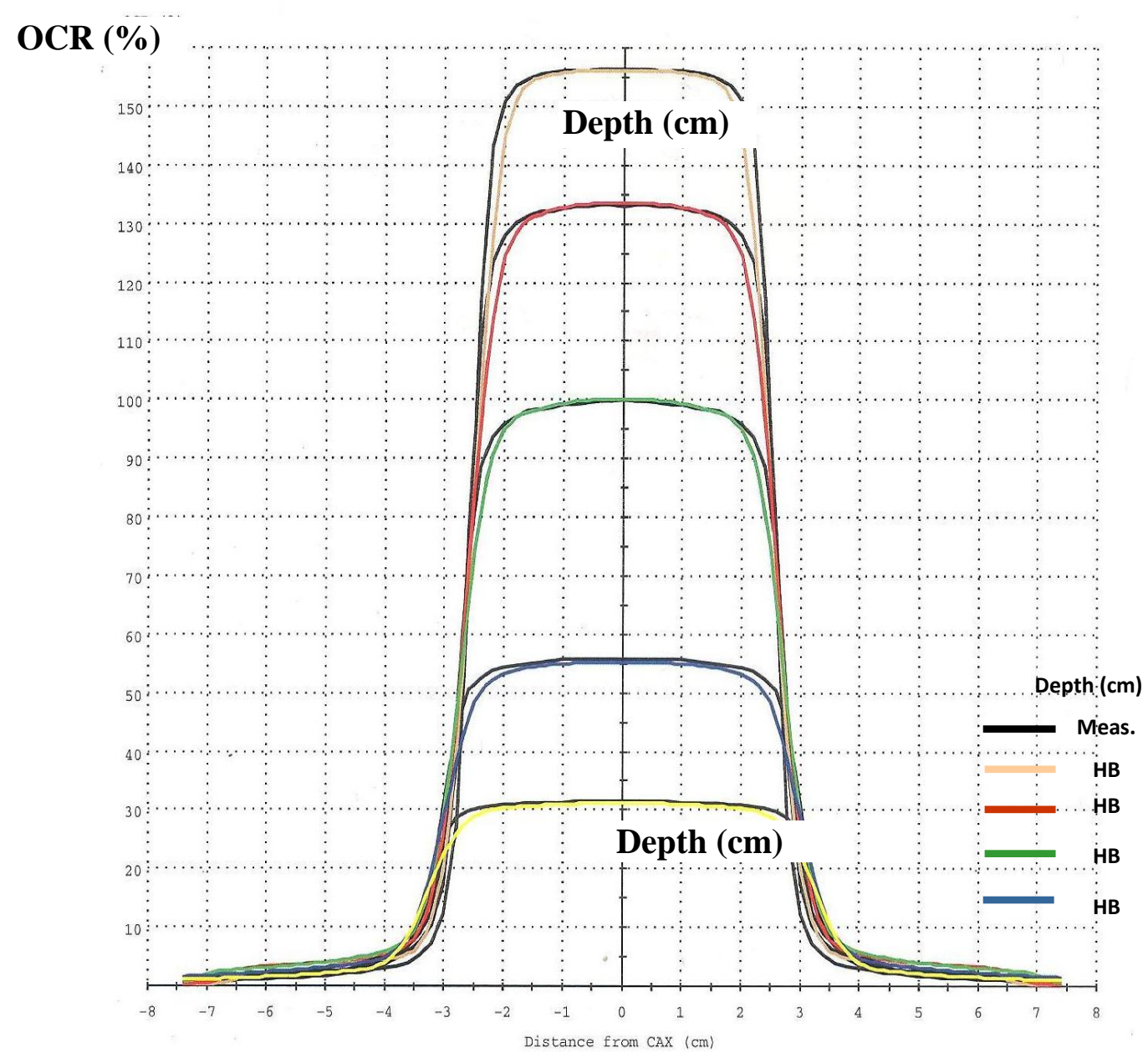

Fig. 3.3 a: In-plane profile for $5 \times 5 \mathrm{~cm}^{2}$ field size 


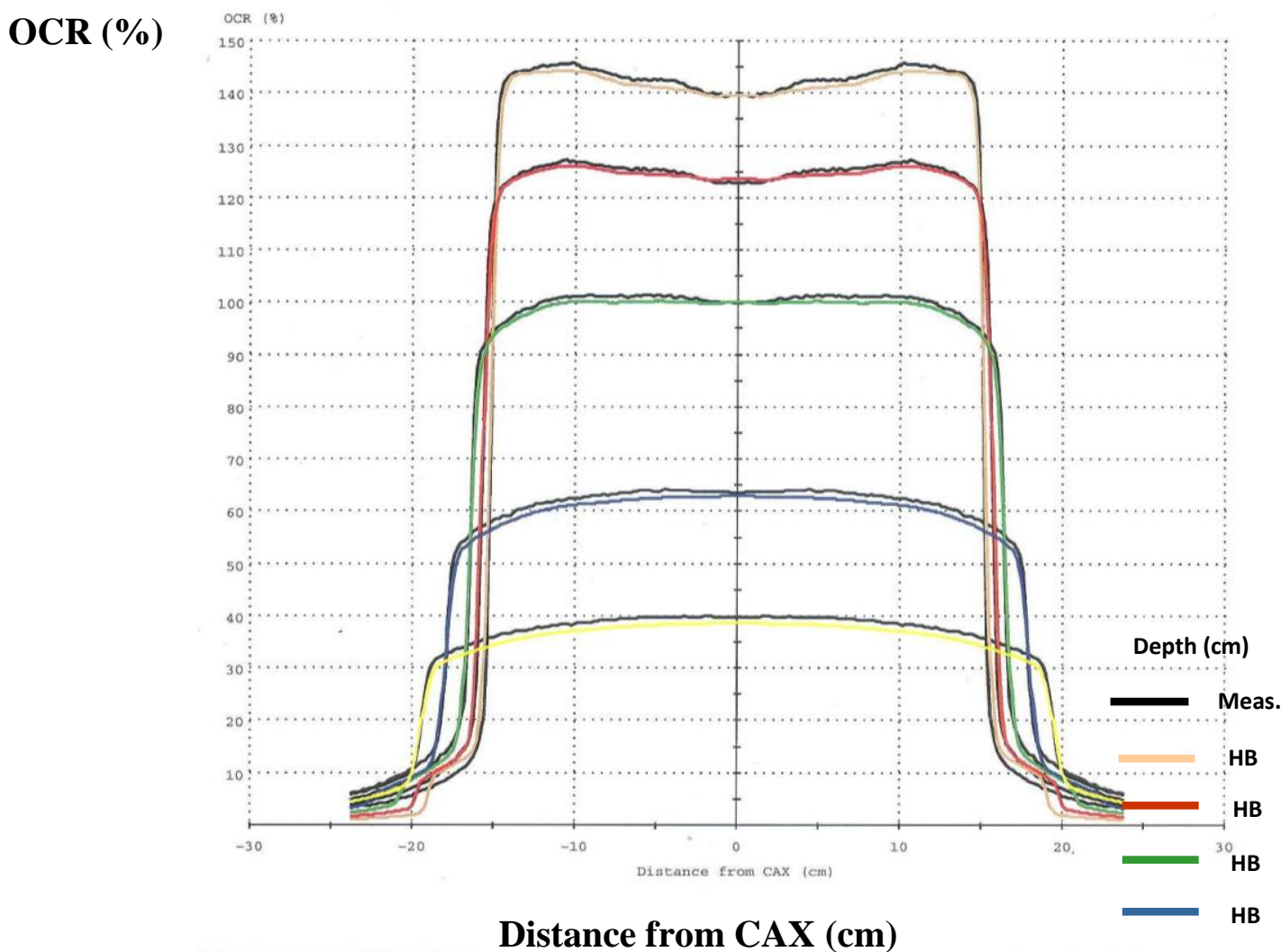

Fig. $3.3 \mathrm{~b}$ : In-plane profile for $30 \times 30 \mathrm{~cm}^{2}$ field size

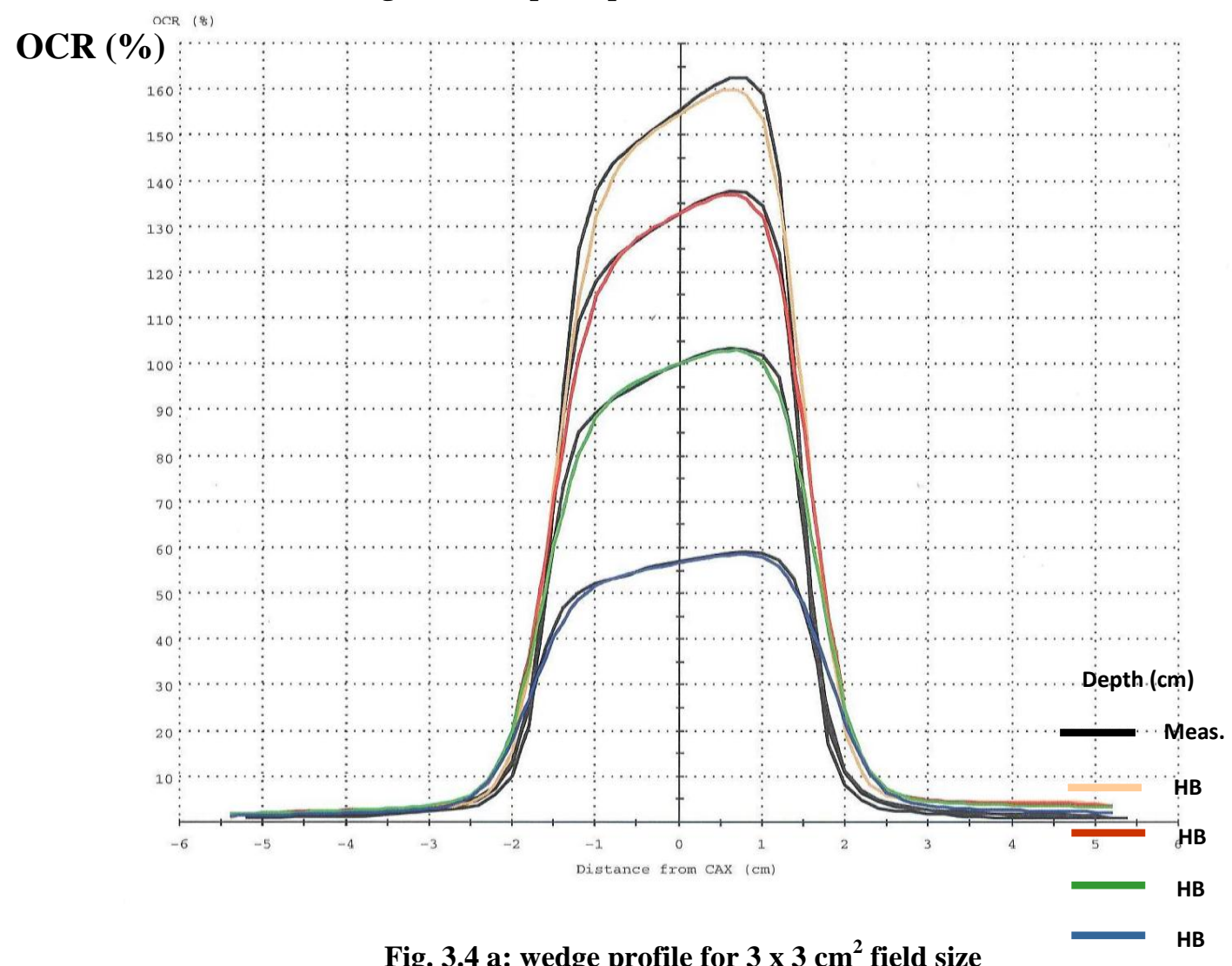




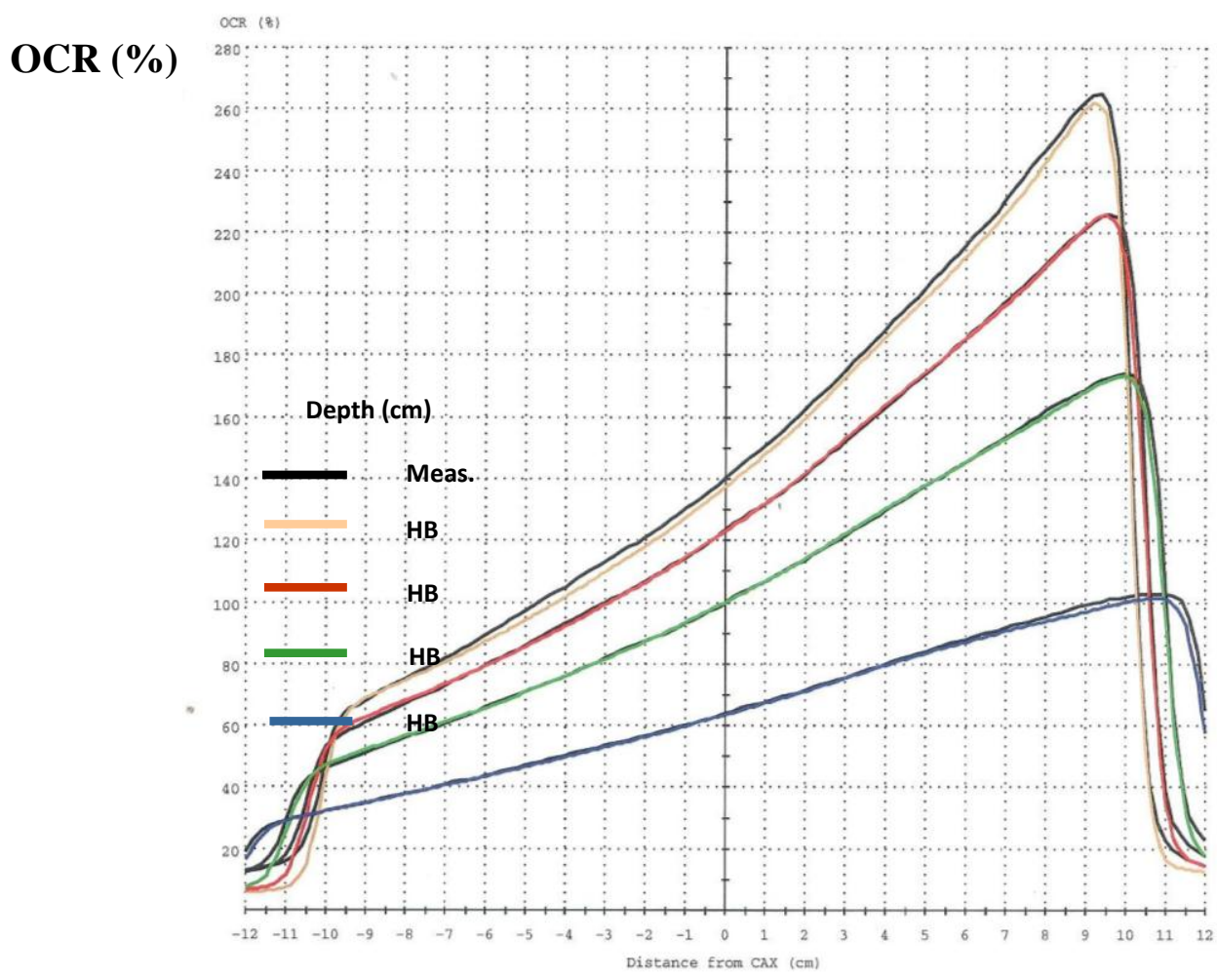

Fig. 3.4 b: wedge profile for $20 \times 20 \mathrm{~cm}^{2}$ field size

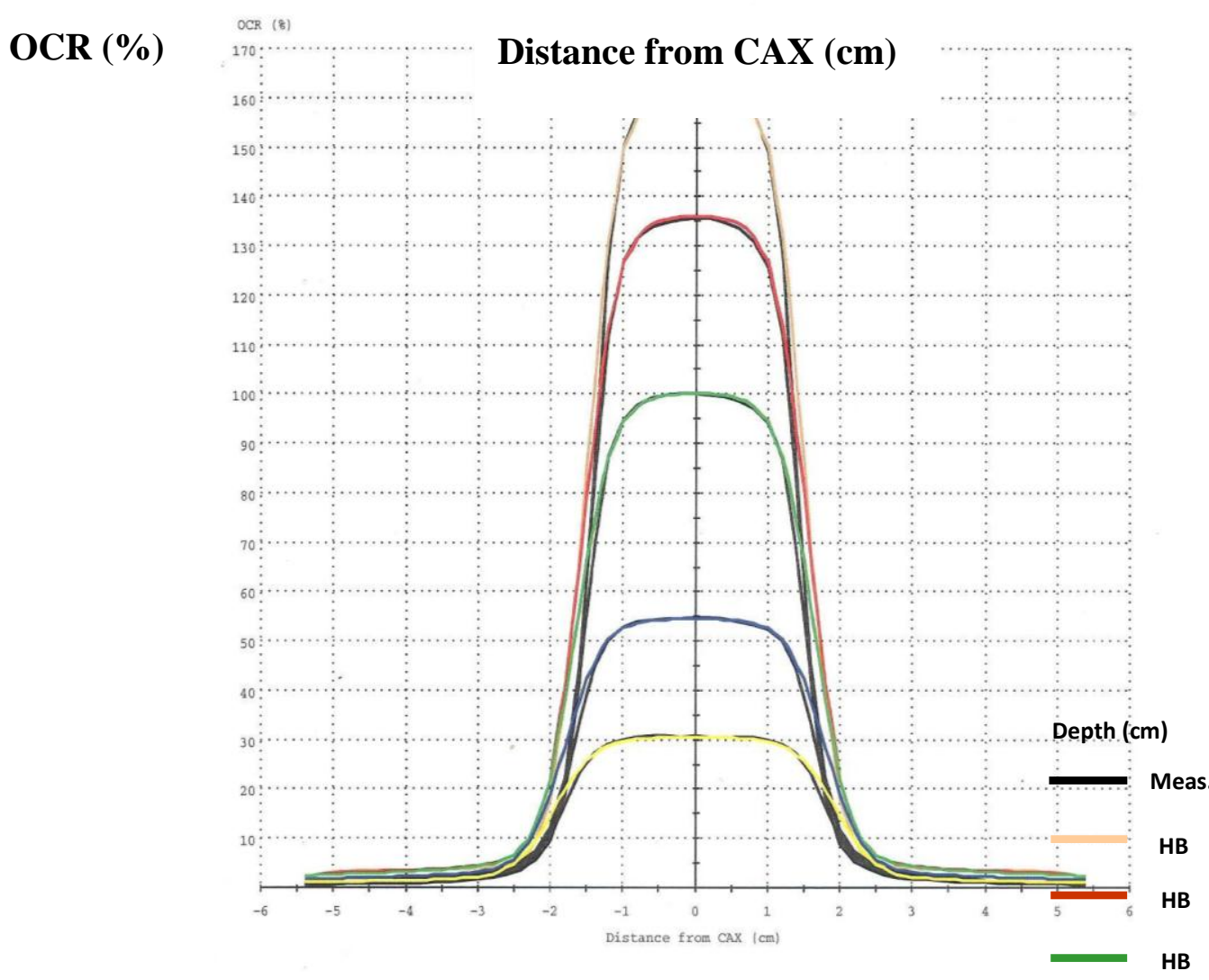

Fig. 3.5 a: Cross-plane Profiles for $3 \times 3 \mathrm{~cm}^{2}$ field sizes $\quad$ HB 


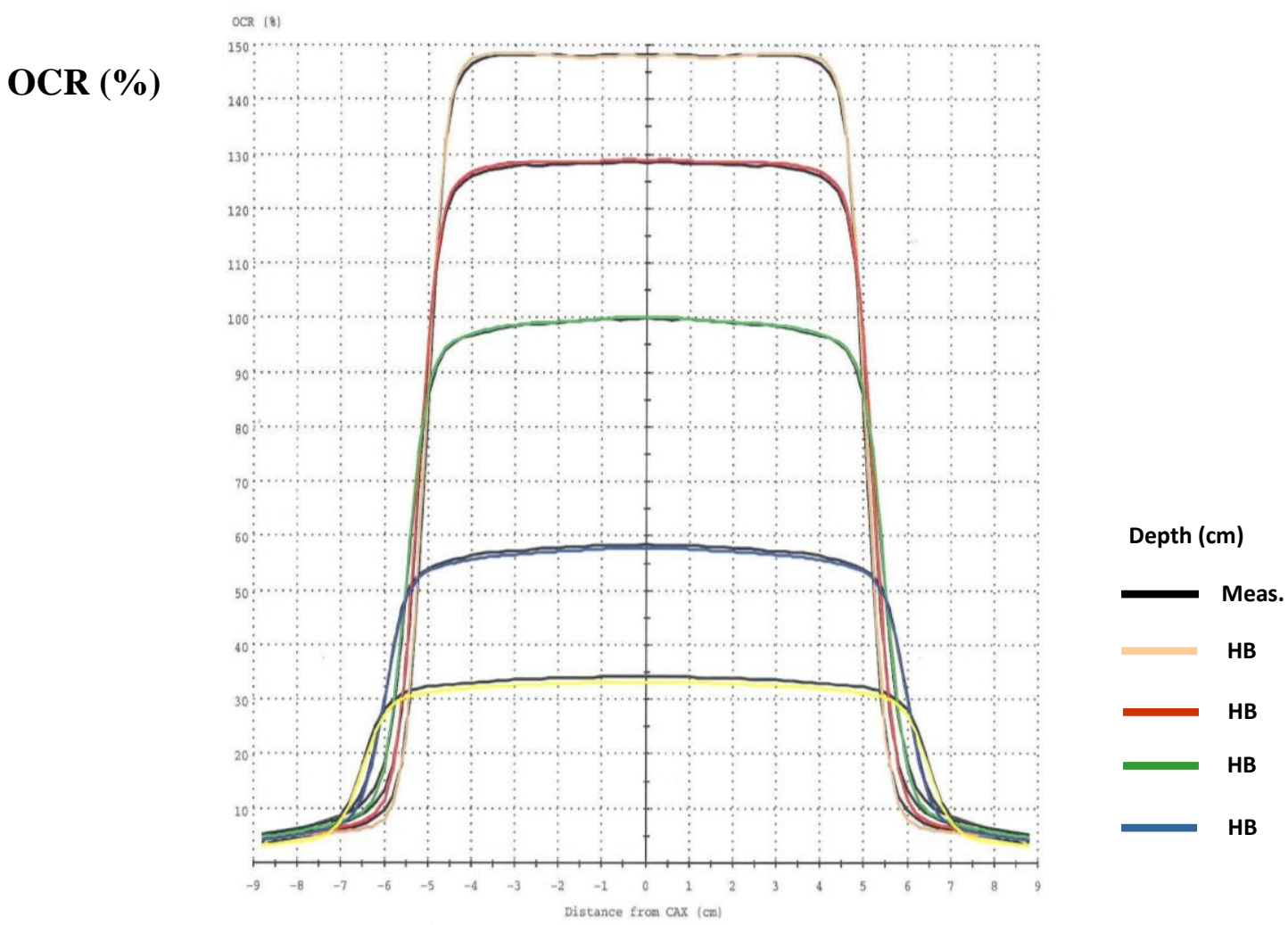

Fig. 3.5 b: Cross-plane Profiles for $10 \times 10 \mathrm{~cm}^{2}$ field size Scanned data for $18 \mathrm{MeV}$ photon beam

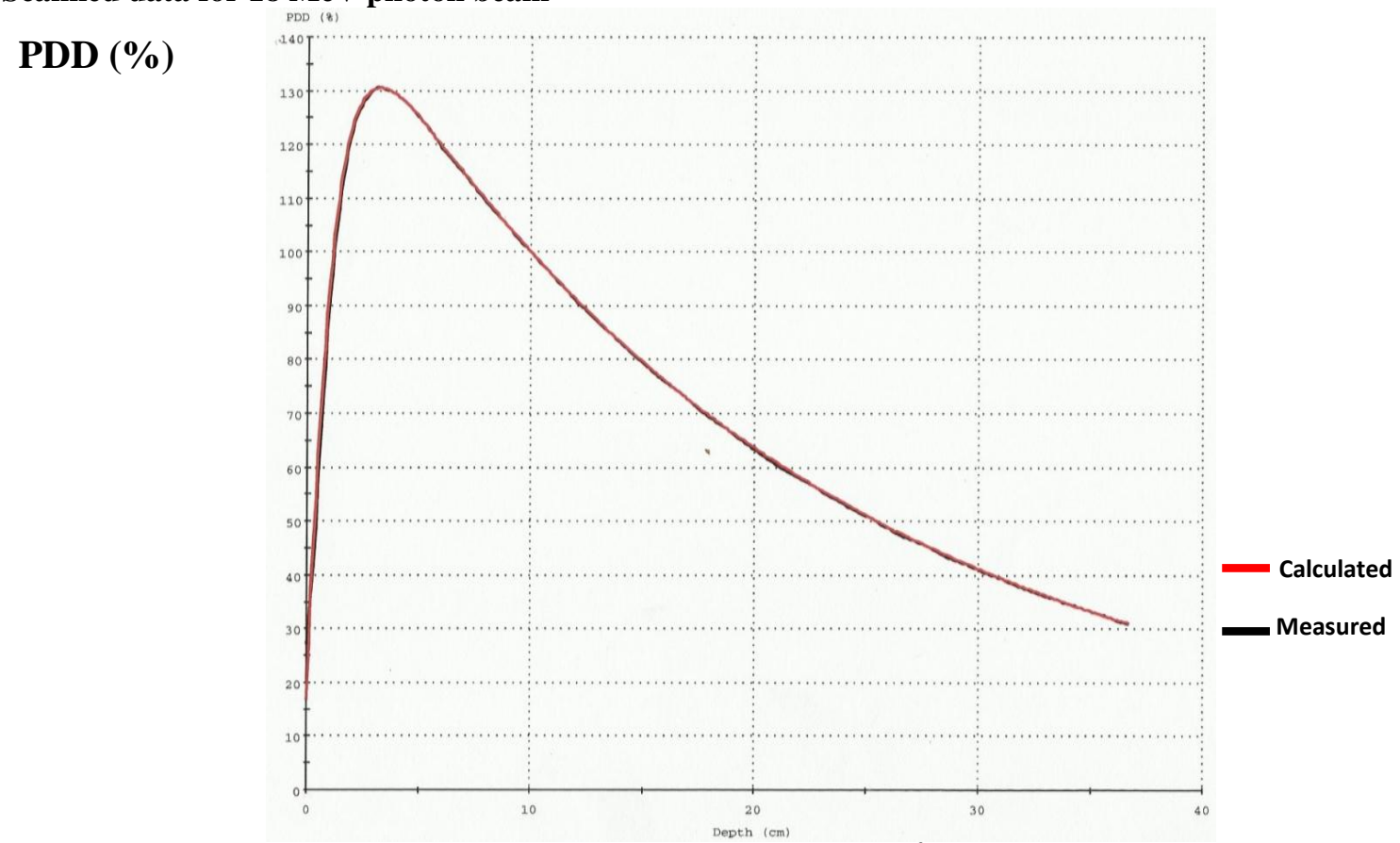

Fig. 3.6a: $18 \mathrm{MeV}$ PDD for $3 \times 3 \mathrm{~cm}^{2}$ field size.

Distance from CAX $(\mathrm{cm})$ 
PDD (\%)

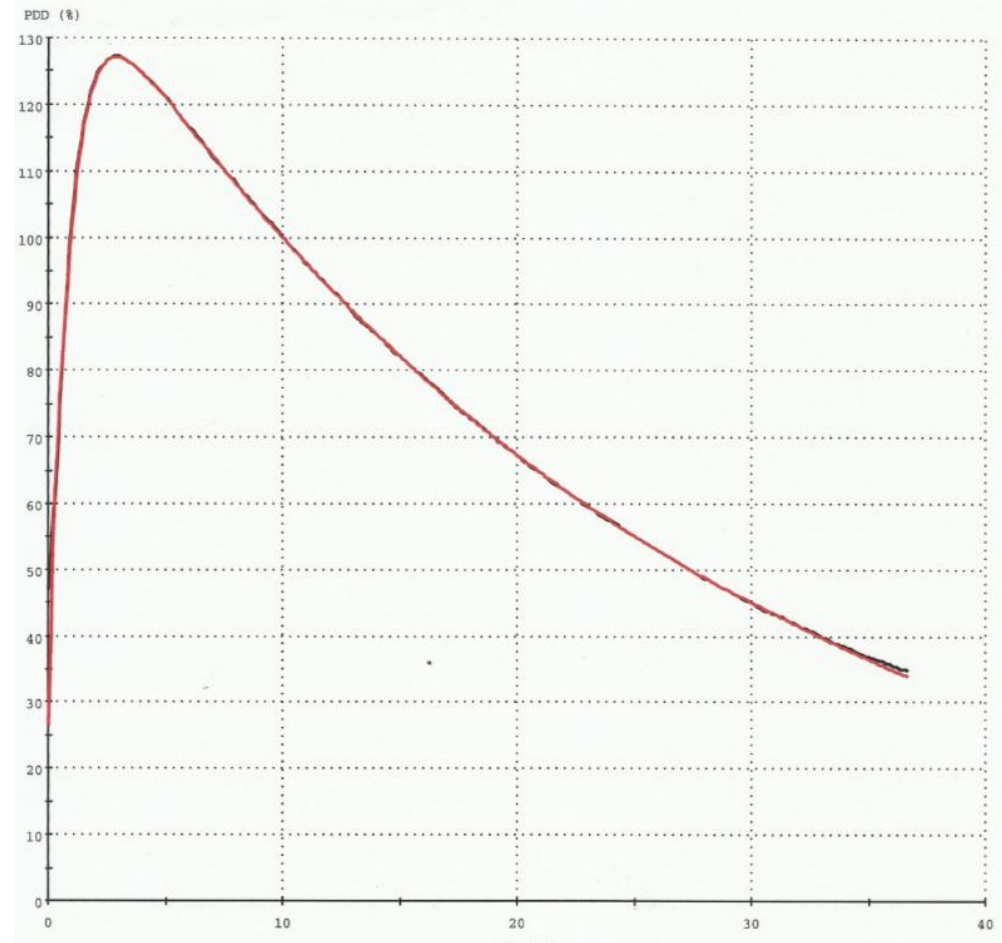

Fig. 3.6b: $18 \mathrm{MeV}$ PDD for $15 \times 15 \mathrm{~cm}^{2}$ field size.

PDD (\%)

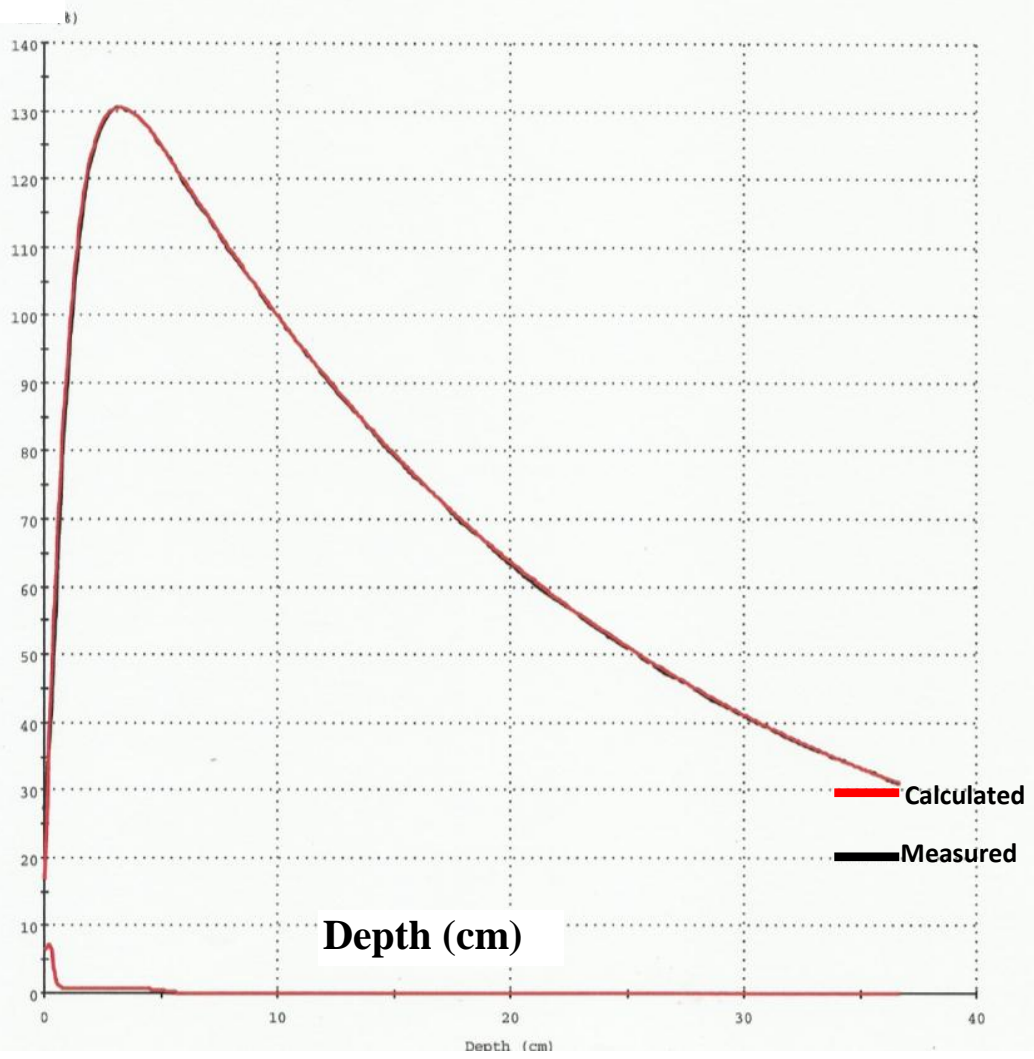

Region of electron contamination

Fig. 3.7a: $18 \mathrm{MeV}$ PDD for $3 \times 3 \mathrm{~cm}^{2}$ field size snuwny enect un enecuun cuntanmanun. 
PDD $(\%)$

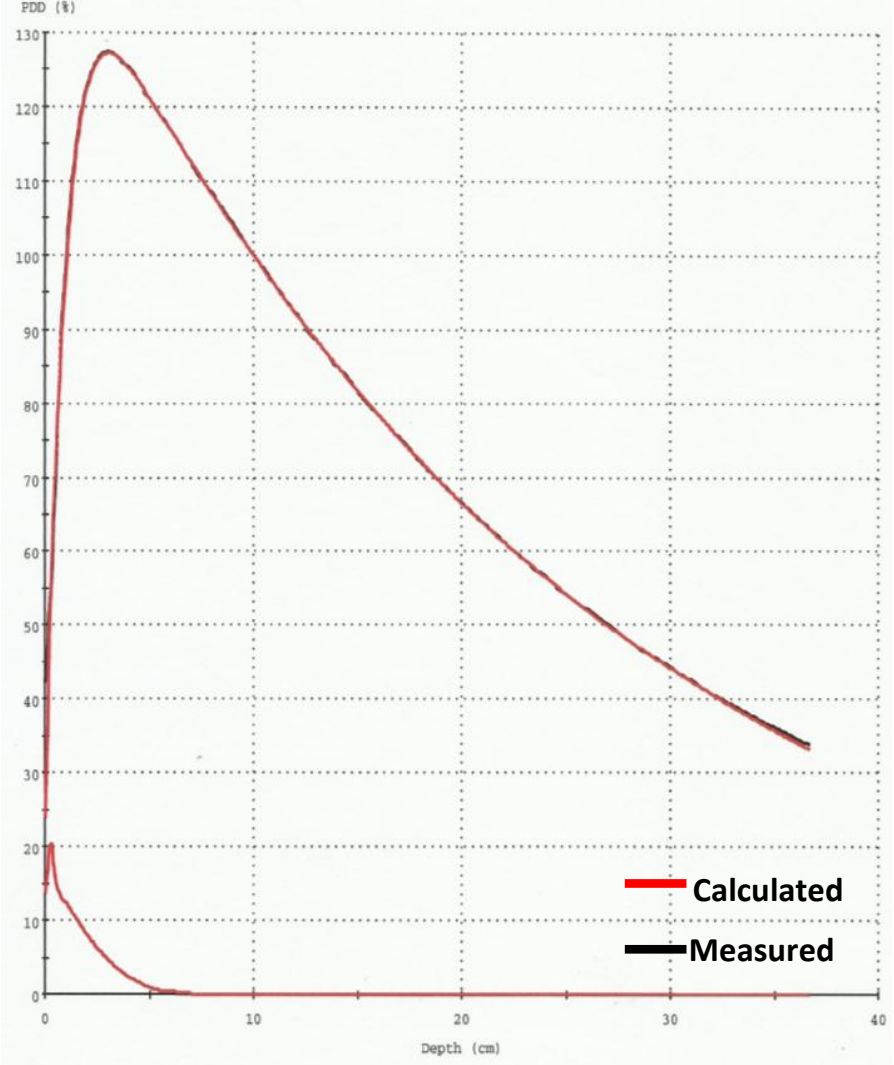

Region of electron contamination

Fig. 3.7b: $18 \mathrm{MeV}$ PDD for 12 x $12 \mathrm{~cm}^{2}$ field size showing effect of electron contamination.

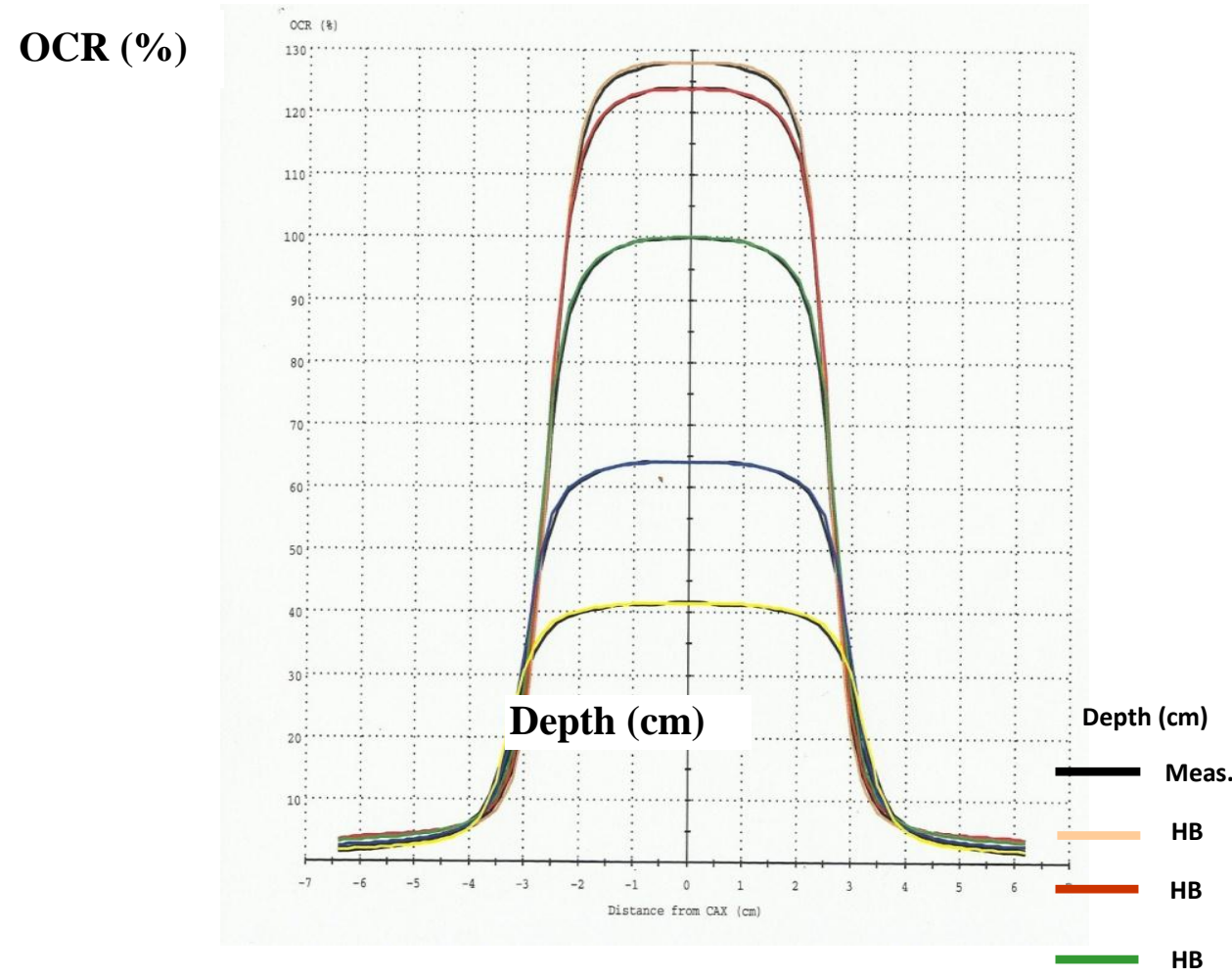

Fig. 3.8a: $18 \mathrm{MeV}$ Crossplane profile for $5 \times 5 \mathrm{~cm}^{2}$ field size нв 
OCR $(\%)$

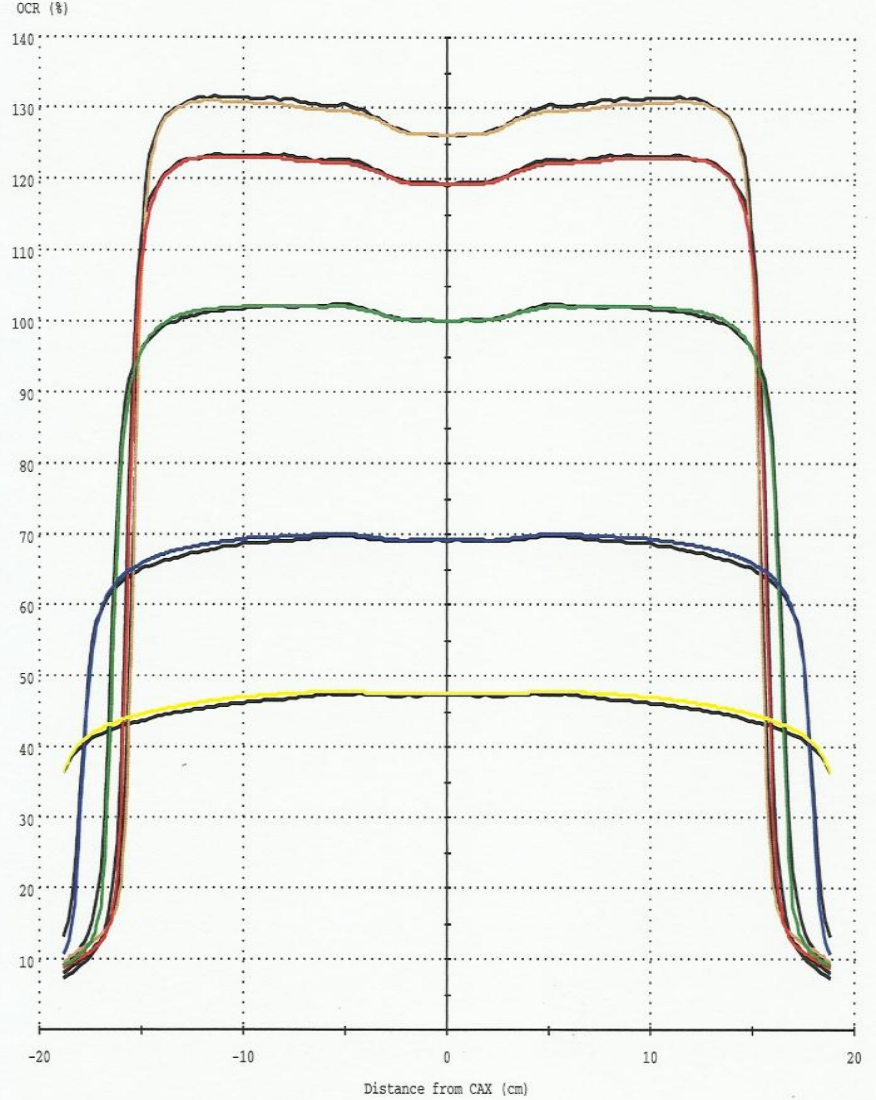

Depth (cm)

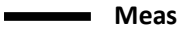

Fig. 3.8b: $18 \mathrm{MeV}$ Crossplane profile for $30 \times 30 \mathrm{~cm}^{2}$ field size.
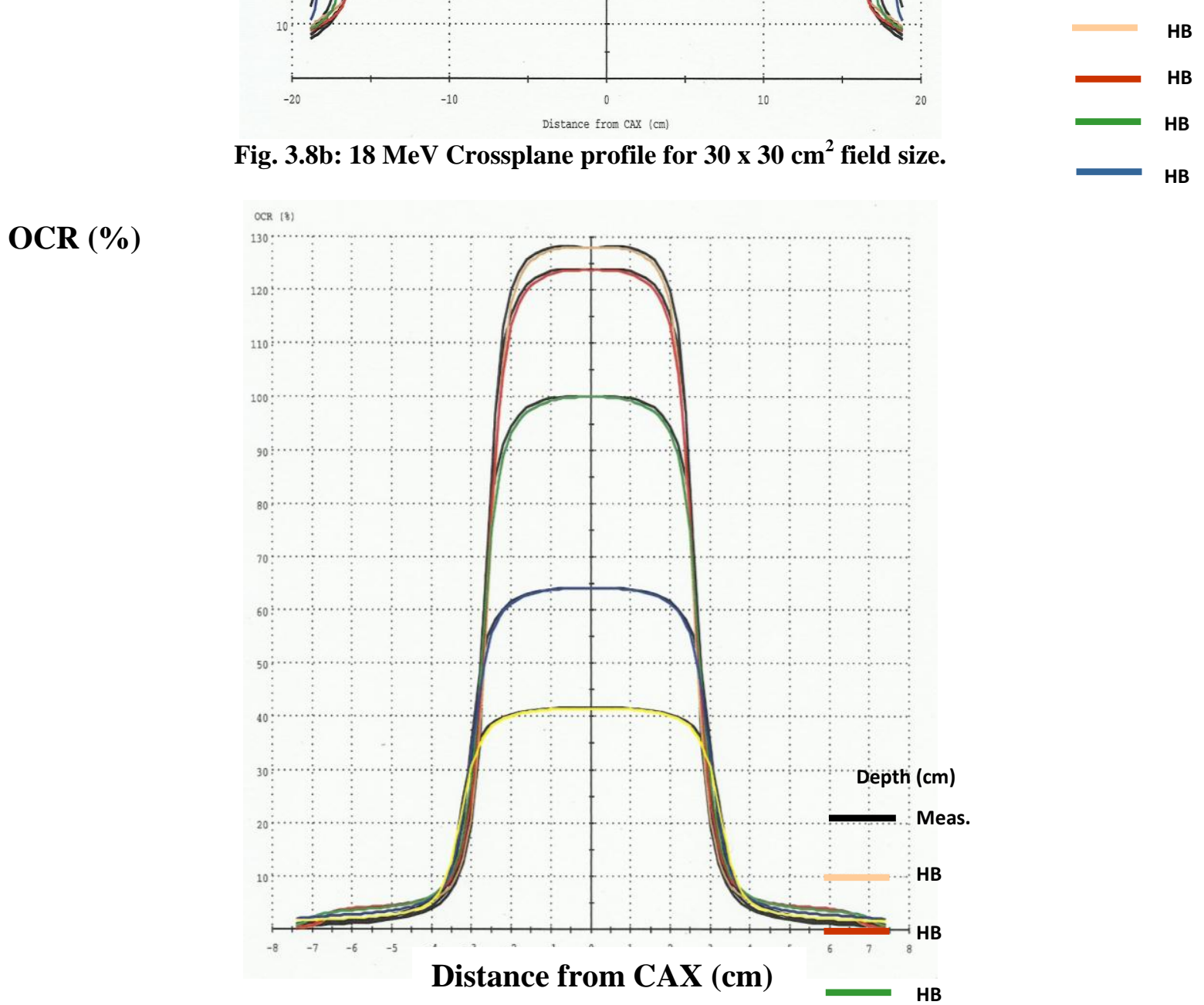

Fig. 3.9a: $18 \mathrm{MeV}$ Inplane profile for $5 \times 5 \mathrm{~cm}^{-}$field size.

HB 


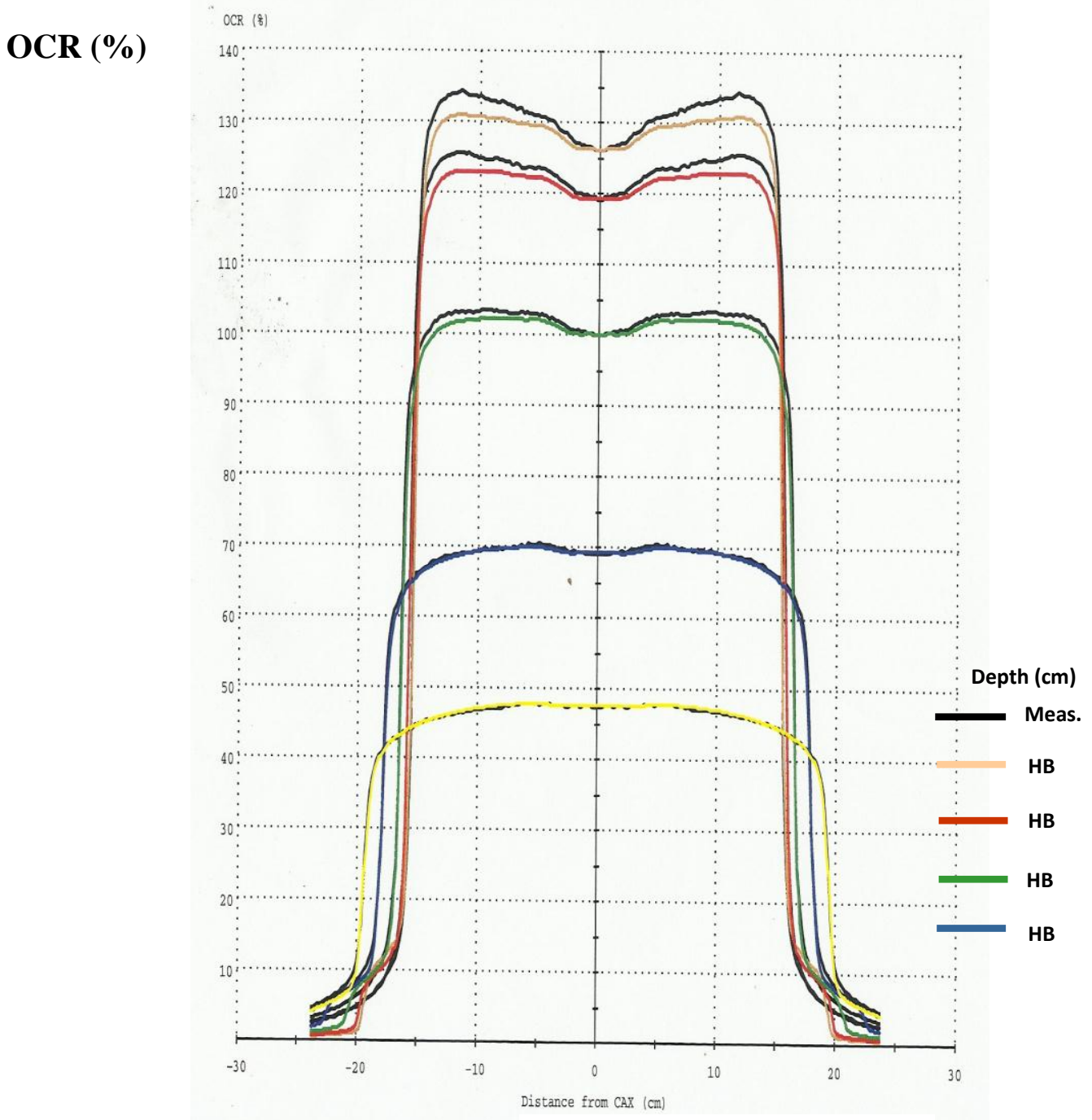

Distance from CAX $(\mathrm{cm})$

Fig. 3.9b: $18 \mathrm{MeV}$ Inplane profile for $30 \times 30 \mathrm{~cm}^{-}$field size.

\section{Discussion And Conclusion}

The results of the normal PDDs determined at reference depth of $10 \mathrm{~cm}$ for different field sizes against the calculated PDDs for the $6 \mathrm{MeV}$ photon beam are represented in figs.3.1(a) and (b). The calculated PDDs completely overlaps the measured PDDs as observed in fig.3.1(a) for the small field size while there is a shift in the PDD tail for large field size as observed in fig. 3.1(b). However the shift is negligible. For the wedge PDDs, the shift between the measured PDDs and the calculated occurs at the $\mathrm{D}_{\max }$ region and it increases with increase in field size as observed in figs 3.2(a) and (b). This may be due to inability of the algorithm to model the fluence calculation for wedge [7-8]. The results of the normal PPDs for the $18 \mathrm{MeV}$ photon beam which are presented in figs. 3.6(a) and (b) follows a similar pattern to those of the $6 \mathrm{MeV}$ photon beam. The calculated PDDs completely overlap the measured PDDs. Electron contamination has been shown to increase in larger field sizes and higher photon energy [10], this is evident in the result of the $18 \mathrm{MeV}$ photon beam presented in figs. 3.7 (a) and (b). The electron contamination in the smaller field size $\left(3 \times 3 \mathrm{~cm}^{2}\right)$ PDD in fig. 3.7(a) is much lesser compared to that of the $12 \times 12 \mathrm{~cm}^{2} \mathrm{PDD}$, this is because electron contamination is mostly caused by the components (i.e. flattening filter, collimators, monitor chamber, etc) in the head of the LINAC. When collimator opening is decrease (i.e. small field size), the electron contamination also decreases as part of the electron source would have been shielded by the collimator blocks.

The variation of dose occurring on a line perpendicular to the central beam axis at a certain depth is known as the beam profile. It represents how dose is altered at points away from the central beam axis. There is also a slight shift between calculated in-plane profiles as shown in figs. 3.3(a) and (b). The calculated wedge profile for the $6 \mathrm{MeV}$ photon beam have a slight shift at the shoulder as observed in figs. 3.4(a) and (b) 
compared to the measured one and this decreases with increase in field size unlike the PDDs. The cross-plane profiles also follow a similar pattern to the in-planes as shown in figs. 3.5 (a) and (b) for the $6 \mathrm{MeV}$ photon beam. The $18 \mathrm{MeV}$ photon beam cross-plane and in-plane profiles presented in figs. 3.8(a) and (b) and 3.9 (a) and (b) also follows similar pattern to those of the $6 \mathrm{MeV}$ photon beam. However, large deviations between calculated and measured are observed in the profiles of the larger field size $\left(30 \times 30 \mathrm{~cm}^{2}\right)$, this is of less concern since most clinical field sizes are lesser. Generally, there is an improvement in the tail region of all the calculated profiles; the region that determines the penumbra of the beam. The penumbra is the region of rapid dose fall off located at the edge of a beam. It is usually considered to be the part of the dose that lies between 20 and $80 \%$ of the central axis dose. The slight shift between calculated and measured PDDs and profiles is negligible.

Generally, there is a good agreement between the measured beam data and the calculated ones as shown in the results using the algorithm. This algorithm can be implemented as an in-house algorithm for modelling photon beam data and also as an independent quality assurance tool for checking the accuracy of clinical TPS algorithms with regards to beam data modelling during commissioning and annual QA checks.

\section{References}

[1]. Podgorsak EB. Radiation Oncology Physics: A handbook for Teachers and Students. Vienna: IAEA publication. 2005.

[2]. Van Dyk J, Barnett RB, Cygler JE, Shragge PC. "Commissioning and quality assurance of treatment planning computers." Int. J. Radiat. Oncol. Biol. Phys. 1993; 26:261-273.

[3]. Van Dyk J. “Quality Assurance.” In Treatment Planning in Radiation Oncology. Khan FM, Potish RA (Eds.). (Baltimore, MD: Williams and Wilkins). 1997;123-146.

[4]. Shaw JE. (Ed.) "A Guide to Commissioning and Quality Control of Treatment Planning Systems.” The Institution of Physics and Engineering in Medicine and Biology. 1994.

[5]. Fraass BA, Doppke K, Hunt M, Kutcher G, Starkschall G, Stern R, Van Dyk J. "American Association of Physicists in Medicine Radiation Therapy Committee Task Group 53: Quality Assurance for Clinical Radiotherapy Treatment Planning.” Med. Phys. $1998 ; 25: 1773-1829$

[6]. Fraass BA. "Quality Assurance for 3-D Treatment Planning." In Teletherapy: Present and Future. Palta J, Mackie TR (Eds.). Madison: Advanced Medical Publishing. 1996;253-318.

[7]. Mackie TR, Scrimger JW, Battista JJ. A convolution method of calculating dose for 15 MV x-rays. Med Phys. 1985;12:188-96.

[8]. Boyer AL, Zhu Y, Wang L, Francois P. "Fast Fourier transform convolution calculations of x-ray isodose distributions in inhomogeneous media,’’ Med. Phys. 1989;16:248-253.

[9]. Sjogren R, Karlsson M. 1996. Electron contamination in clinical high energy photon beams. Med. Phys. 23: 1873-81.

[10]. Lewis EE, Miller WF. 1984. "Computational methods of neutron transport”, New York Wiley publication.

[11]. Wareing TA, McGhee JM, Morel JE, Pautz SD. 2001. Discontinuous Finite Element Sn Methods on Three-Dimensional Unstructured Grids. Nucl. Sci. Engr., 138:2.

[12]. Wareing TA, Morel JE, McGheeJM. 2000. Coupled Electron-Proton Transport Methods on 3-D Unstructured Grids, Trans Am. Nucl. Soc.83.

[13]. Siebers JV, Keall PJ, Nahum AE, Mohan R. 2000. Converting absorbed dose to medium to absorbed dose to water for Monte Carlo based photon beam dose calculations. Phys. Med. Biol. 45:983-995.

[14]. CMS Xio Beam Modelling Guide. 2008. Xio version 4.62 treatment planning system. Stockholm: Eleckta software publication.

[15]. Vassiliev ON, Wareing TA, McGhee J, Failia G. 2010. Validation of a new grid-based Bolzmann equation solver for dose calculation in radiotherapy with photon beams. Phys. Med. Biol. 55:581-598. 